Revue scientifique sur la conception et l'aménagement de l'espace

\title{
Le plan local d'urbanisme français : un instrument orienté de pédagogie citoyenne du paysage
}

The local urban development plan: An oriented instrument for developing citizen awareness of the landscape

\section{Mathieu Gigot et Arnaud de Lajartre}

\section{(2) OpenEdition}

\section{Journals}

Édition électronique

URL : http://journals.openedition.org/paysage/1063

DOI : $10.4000 /$ paysage. 1063

ISSN : 1969-6124

Éditeur :

École nationale supérieure du paysage de Versailles-Marseille, Institut national des sciences appliquées Centre Val de Loire - École de la nature et du paysage, École nationale supérieure d'architecture et de paysage de Bordeaux, École nationale supérieure d'architecture et de paysage de Lille, Agrocampus Angers

\section{Référence électronique}

Mathieu Gigot et Arnaud de Lajartre, «Le plan local d'urbanisme français : un instrument orienté de pédagogie citoyenne du paysage », Projets de paysage [En ligne], 18| 2018, mis en ligne le 04 juillet 2018, consulté le 28 novembre 2019. URL : http://journals.openedition.org/paysage/1063 ; DOI : 10.4000/paysage.1063

Ce document a été généré automatiquement le 28 novembre 2019.

Projets de paysage 


\section{Le plan local d'urbanisme français : un instrument orienté de pédagogie citoyenne du paysage}

The local urban development plan: An oriented instrument for developing citizen awareness of the landscape

Mathieu Gigot et Arnaud de Lajartre

1 Si le paysage peut apparaître comme un « angle mort » des dernières lois adoptées en matière d'environnement et d'urbanisme, cela s'explique en partie par le fait qu'il est déjà largement intégré au système juridique français, en particulier au travers de l'un des outils de synthèse des enjeux d'urbanisme, d'environnement et de patrimoine qu'est le plan local d'urbanisme (PLU), ou au fil du renforcement des intercommunalités le PLUi. Les PLU comportent désormais de nombreux passages et dispositions réglementaires dédiés au paysage. À ce titre, ils participent de facto à une forme de didactique du paysage, à la fois au titre de leur contenu final imposé aux demandeurs d'autorisations d'urbanisme (habitants, entreprises...), mais également en amont à travers leur procédure d'élaboration et de concertation favorisant par principe les échanges entre acteurs sur le sujet. Ce double constat émerge d'un programme de recherche sur les liens entre PLU et patrimoine ${ }^{1}$, financé par l'Agence nationale de la recherche (ANR), et conduit depuis trois ans par une équipe de chercheurs et d'enseignants-chercheurs rassemblant juristes, géographes, sociologues et architectes. 
Figure 1. Le programme « PLU Patrimonial »

- Un programme de septembre 2015 à septembre 2019, rassemblant l'EA-CIB (Université d'Angers, I'UMR CITERES-CNRS (Université de Tours), l'EVS (École d'Architecture de Lyon) et l'EA-DCT (Université Louis Lumière Lyon 2).

- Une analyse méthodologique et juridique (grille d’analyse) d'un échantillonnage de PLU communaux (ou de secteurs communaux de PLU intercommunaux) ;

- Un ensemble d'entretiens avec les acteurs de ces documents et processus de patrimonialisation (services des collectivités, ABF, associations, élus, agences d'urbanisme, etc.);

- Des territoires étudiés essentiellement urbains : l'échantillonnage scientifique de référence soumis à l'ANR repose sur les 20 plus grandes villes de France ;

- Élargissement à des villes de petite ou moyenne taille, grâce à des partenariats avec certains masters associés au programme de recherches.

- Au total, 35 PLU finement radiographiés sous l'angle du patrimoine, mais aussi du paysage, incluant très peu de territoires purement ruraux souvent dépourvus de PLU (près de 10.000 (toutes) petites communes seulement restent soumises au RNU et près de 4.000 aux cartes communales, même si ce dernier chiffre diminue dans le cadre du passage aux PLUi. Néanmoins l'ensemble regroupe à peine 6 millions d'habitants, alors que plus de 60 millions sont soumis à des PLU ou PLUi, dont par exemple la quasi-totalité des communes littorales).

L'étendue géographique du programme de recherches comme sa temporalité par rapport à celle des PLU n'ont pas permis, sauf proximité géographique du chercheur, de suivre finement les procédures d'élaboration des PLU étudiés, en participant par exemple à l'ensemble des réunions de travail des services compétents ou de concertation avec la population et les associations. II ne sera donc pas possible dans cet article d'assurer la traçabilité la plus fine de la notion de paysage portée par tel ou tel document. De plus, cet article repose sur la restitution de l'analyse de données d'entretiens ou de pièces de PLU(i) issues de plusieurs dizaines de documents qui ne pourront à ce titre être citées in extenso.

2 La recherche d'occurrences (entre autres méthodes d'analyse scientifique) du mot «patrimoine » (et de ses qualificatifs associés) mais aussi du mot «paysage » montre que si tout ce qui relève du paysage dans le PLU ne s'inscrit pas systématiquement dans la thématique du patrimoine, en sens inverse, une partie de ce dernier s'inscrit dans le champ du paysage. Dans cette optique, la lecture de l'intégralité des dossiers de PLU a permis de mettre en évidence la diversité des acceptions du paysage retenues autant dans une optique de protection (les paysages patrimoniaux) que dans une perspective de gestion ou d'aménagement (le paysage cadre de vie, ou le paysage à reconquérir des entrées de ville et des franges urbaines). Les notions de paysage et de patrimoine qui seront mobilisées dans cet article sont donc avant tout celles des acteurs, c'est-à-dire des collectivités locales et des établissements publics compétents en matière de planification urbaine, ainsi que des structures publiques ou privées contribuant à la fabrique des PLU: bureaux d'études, agences d'urbanisme, Conseils d'architecture, d'urbanisme et de l'environnement (CAUE), associations locales, etc.

3 Sur la question de la pédagogie du/au paysage par le PLU, deux enseignements principaux se dégagent des terrains et documents étudiés dans le programme de recherche PLU patrimonial. D'une part, en intégrant le paysage au sein de la planification urbaine, les PLU génèrent un contexte territorial et une situation sociale favorisant l'apprentissage citoyen - parfois même institutionnel - sur le paysage : pour un habitant devant le consulter aux fins d'un permis de construire ou pour un service instructeur chargé de l'appliquer, un échange aura lieu sur la conception du paysage. L'élaboration puis l'ouverture d'une PLU offrent donc un cadre pédagogique impliquant a minima une sensibilisation - parfois un début de formation - des citoyens en matière de paysage. La situation d'apprentissage ainsi créée place en quelque sorte le citoyen en situation d'élève (Zask, 2001), la découverte de la notion se faisant souvent lors d'une demande de permis de construire. D'autre part, cette didactique - par l'action - oriente 
sur le fond les représentations du paysage que s'en feront habitants et acteurs; en effet, le PLU distille tout au long du dossier une acception plurielle du paysage, allant d'une approche très large de ce dernier - à l'instar de celle qu'évoque la Convention européenne du paysage (CEP $)^{2}$ - jusqu'à son découpage en une variété d'objets relevant schématiquement tantôt du patrimoine urbain, tantôt du paysage naturel et souvent végétal, en tout cas tangible. Du rapport de présentation au règlement, l'analyse de nombreux PLU révèle ainsi un glissement sémantique autour du mot "paysage » influençant inévitablement les représentations que peuvent s'en faire les habitants, et même les élus et les services.

4 Les PLU participent ainsi d'une pédagogie du paysage par la pratique lors des procédures d'adoption et de mise en œuvre de la planification urbaine, mais d'une pédagogie orientée par le vocabulaire retenu pour évoquer le paysage dans les différentes pièces du PLU.

\section{PLU et pédagogie du paysage ex ante et ex post}

5 La vie d'un PLU offre plusieurs temps d'échanges possibles autour du thème du paysage, permettant de pratiquer à cette occasion une pédagogie protéiforme : le $1^{\mathrm{er}}$ temps est celui des procédures d'élaboration et de concertation citoyenne de la planification. Un second temps correspond à sa mise en œuvre après approbation, imposant alors que les services territoriaux forment les habitants à cet enjeu, en ayant souvent eux-mêmes dû se former à cette question par des échanges interservices.

\section{La « formation » au paysage pendant l'élaboration du PLU}

6 Depuis une dizaine d'années, le passage obligé au PLU (loi SRU) puis au PLUi (loi ALUR) rénove profondément la planification urbaine française (Traoré, 2014, p. 350). Cette étape importante dans le droit de l'urbanisme favorise une forme de pédagogie du paysage, et ce pour deux raisons. D'une part, le paysage est l'un des items listés par le code de l'urbanisme dans le cadre des objectifs imposés aux SCoT et PLUi. L'article L. 101-2 évoque en effet aussi bien «la protection des paysages» (L. 101-2 - $6^{\circ}$ ), en particulier des "paysages naturels» (L. 101-2 - $1^{\circ}$-c) que la "qualité urbaine, architecturale et paysagère, notamment des entrées de ville» (L. 101-2 - $\left.2^{\circ}\right)$. D'autre part, il ne s'agit là que de l'une des conséquences de la CEP qui impose d'intégrer la question du paysage dans les politiques publiques. Les politiques urbaines y répondent en particulier au travers des documents réglementaires, injectant du paysage dans une multitude de thématiques dont ils doivent se saisir. En effet, l'article L. 101-2 impose aux collectivités locales en charge de la planification urbaine de prendre en compte en les articulant les nombreux enjeux qui font la globalité d'un territoire: logements, équipements publics et privés, activités économiques, agriculture, environnement, changement climatique, etc. Le paysage ne constitue, par conséquent, qu'un objectif parmi d'autres, mais il est en même temps un objectif particulier : il est certainement l'un des rares à pouvoir "traverser " les autres, puisque, au regard de la définition même du paysage donnée par la CEP, désormais inscrite à l'article L. 350-1 A du code de l'environnement, tout peut être considéré comme paysage. Résultant de l'action de "facteurs naturels ou humains et de leurs interrelations dynamiques", le paysage recouvre l'ensemble des caractéristiques géophysiques et socioéconomiques d'un 
territoire ; il se nourrit par conséquent de l'ensemble des items énumérés par l'article L. 101-2. L'élaboration d'un PLU offre donc toutes les conditions pour parler du paysage; ce dernier se trouve d'ailleurs souvent instrumentalisé comme support facilement partagé de projet territorial (Labat et Donadieu, 2013, p. 44). Par son accessibilité linguistique, le paysage (souvent celui des vacances ou des grands sites qui fait partie du vocabulaire de tout un chacun), est un mot facile pour rassembler habitants et acteurs du territoire autour de la table, et mettre en débat ensuite les enjeux sociaux, économiques et environnementaux. Le ministère en charge du logement ne s'y est pas trompé (ou certaines DREAL), en retenant le paysage comme l'un des items qui permet d'activer la dynamique des clubs $\mathrm{PLUi}^{3}$ et de promouvoir les bonnes pratiques des collectivités pionnières en la matière. Sur nos terrains d'étude, dans le cadre de l'élaboration de son PLUi, la communauté de communes du Grand Chambord (Loir-et-Cher) a pris le parti de prendre appui sur le paysage pour rassembler élus et habitants autour d'un projet de territoire commun.

7 Ces débats relatifs au paysage commencent dès les échanges entre maitrise d'ouvrage et maîtrise d'œuvre ; l'élaboration d'un PLUi peut se faire en régie, mais elle est le plus souvent déléguée à un bureau d'études, à un cabinet d'urbanistes ou parfois à une agence d'urbanisme publique. Le choix du prestataire n'est pas anodin : il aura une influence sur la pédagogie du paysage qui se jouera dans un premier temps - et à un premier niveau - entre l'équipe en charge de l'élaboration du document et les élus. Ces derniers n'ont pas souvent de connaissances théoriques sur le sujet ni de culture du paysage. L'expertise en la matière d'élus comme Christophe Degruelle (président d'Agglopolys, Loir-et-Cher) ou Sébastien Giorgis (adjoint au maire d'Avignon, Vaucluse) reste exceptionnelle, le premier étant enseignant à l'École de la nature et du paysage de Blois, et le second paysagiste-conseil et président en 2014 de l'Association des paysagistes-conseils de l'État. En revanche, pour la plupart des élus comme des habitants, un apprentissage de la dimension polysémique du paysage s'avère souvent nécessaire ; elle est réalisée par le maître d'œuvre, pour autant que ce dernier ait luimême des compétences en matière de paysage. Un environnementaliste ou un architecte n'aura pas ipso facto la vision transversale et multiscalaire du paysage portée par d'autres professionnels (par exemple les paysagistes) et entérinée par la CEP ; le premier abordant le sujet avant tout au travers de l'écologie du paysage, le second plutôt sous l'angle du paysage en tant qu'ensemble urbain, voire du seul patrimoine bâti. Selon la taille de la structure (publique ou privée) en charge de l'élaboration du PLU, il y aura ou non une personne ressource pour le paysage. Par exemple, à Angers Loire Métropole, dont l'élaboration du PLUi s'est faite en régie, le chargé de mission Trame verte et bleue, géographe de formation, assume également la lecture paysagère du territoire. En revanche les agences d'urbanisme à Tours (ATU) ou à Marseille (AGAM) possèdent un architecte-paysagiste dans ses rangs. Mais dans cette approche du paysage par les techniciens, contribuant ou non à enrichir celle des élus, les jeux d'acteurs en matière de pédagogie paysagère s'arrêtent rarement là. Les consultations obligatoires des personnes publiques associées, le travail partagé (ou imposé, par le biais du contrôle de légalité) avec les services de l'État, l'ouverture volontaire de la procédure d'élaboration à des partenaires comme les CAUE ou certaines associations de protection de l'environnement et du paysage, vont nettement enrichir la construction de la politique paysagère du PLU. Cela nourrit en amont le mot de paysage et la manière de le remplir. La présence d'un paysagiste au CAUE ou dans un Parc naturel régional (PNR), ou la simple intervention d'un paysagiste-conseil à la direction 
départementale des Territoires lors du contrôle de l'État sur le futur PLU, participent fortement de cette pédagogie du paysage que favorise le travail sur la planification urbaine. Ces différents spécialistes du paysage vont amplifier - et même parfois initier - une réflexion sur le champ conceptuel et opérationnel de ce mot-valise et polysémique.

8 Cette pédagogie mutuelle entre techniciens, mais aussi entre techniciens et élus, déborde le champ des services pour s'adresser à la société civile dans toutes ses composantes. En effet l'élaboration du PLU doit répondre à l'obligation de concertation imposée par les articles L. 103-2 à L. 103-6 du code de l'urbanisme. Celui-ci reste relativement laconique sur les objets et le déroulement de cette concertation; il se contente d'affirmer que les modalités de la concertation doivent permettre, "pendant une durée suffisante et selon des moyens adaptés au regard de l'importance et des caractéristiques du projet, au public d'accéder aux informations relatives au projet et aux avis requis par les dispositions législatives ou réglementaires applicables et de formuler des observations et propositions qui sont enregistrées et conservées par l'autorité compétente » (L. 103-4). Ce cadre juridique très général ouvre à la collectivité une variété de "menus de concertation" extrêmement large, allant de simples réunions publiques à un panel d'outils de médiation (expositions, ateliers, balades urbaines, interventions artistiques, sites Internet, etc.) (Davodeau et Toublanc, 2010). Ces outils sont adaptés à la question du paysage dans les PLU : les promenades ou ateliers de terrain offrent des supports pédagogiques pertinents pour permettre d'échanger avec la population sur les différentes facettes de la notion de paysage. L'ouverture d'un atlas photographique de ce qui fait paysage pour les habitants constitue souvent une excellente porte d'entrée pour enclencher une pédagogie citoyenne du paysage : ce qu'un habitant retient comme son paysage, voire un beau paysage, ne l'est pas pour autant pour un habitant d'un autre quartier, voire d'une autre commune quand l'échelle du PLU est intercommunale. Cette pédagogie du paysage se trouve renforcée lorsque les PLU mobilisent ouvertement ce dernier pour lui faire endosser certaines valeurs communes, propres à renforcer la cohésion et l'identité territoriales ; ainsi que cela a déjà été affirmé, le paysage offre les traits d'un sujet englobant permettant, d'une part, de réunir habitants et acteurs autour d'une table et, d'autre part, de mettre en valeur des éléments de partage, voire des biens communs, au sein d'un territoire parfois vaste. On peut d'ailleurs penser que cet enjeu montera en puissance dans le cadre du passage croissant au PLUi : les unités paysagères transcendant souvent de facto les limites communales, elles seront plus facilement transposables dans leur entièreté au sein des PLU intercommunaux. La pédagogie du paysage pratiquée à cette occasion pourra alors servir la fabrique de ces nouveaux territoires de l'urbanisme induits par les nouvelles intercommunalités. Mais, pour que cette pédagogie du paysage, et parfois par le paysage, porte ses fruits, encore faut-il que la concertation pour l'élaboration du PLUi réunisse un échantillonnage suffisant de population; on constate trop souvent la faiblesse de la participation citoyenne lors des temps de concertation relatifs aux projets de planification, ou même parfois aux projets d'aménagement ou d'équipement. L'enjeu politique est pourtant de taille, car une pédagogie du paysage réussie pendant l'élaboration du document d'urbanisme facilitera celle qui s'imposera également pendant sa mise en œuvre. 


\section{La pédagogie du paysage pendant la mise en œuvre du PLU}

Si le temps de la fabrique du PLU se prête à de la formation au paysage des élus, des habitants et parfois des techniciens eux-mêmes, l'entrée en vigueur de la planification urbaine ouvre une seconde "session pédagogique». En effet, la mise en œuvre opérationnelle des dispositions juridiques retenues dans les Orientations d'aménagement et de programmation (OAP) ou dans le règlement va questionner à la fois les administrés, dès qu'ils souhaiteront réaliser des travaux soumis au PLU, mais aussi parfois le service instructeur lui-même, faute d'une compréhension immédiate des enjeux paysagers énoncés dans les documents.

Tout d'abord, lors de chaque demande d'autorisation en droit des sols, le pétitionnaire est censé s'approprier les règles imposées par le PLU pour tous types de travaux. Or, la compréhension d'une telle réglementation pour un administré peu familier des documents juridiques nécessite souvent des explications, pour ne pas dire un "décodeur ». Les nombreuses pièces (figure 2) d'un PLU (Baffert, 2017) représentent logiquement des centaines de pages - voire des milliers pour un PLUi - que l'habitant ne lira pas forcément entièrement; seules vont l'intéresser les pièces juridiquement opposables (règlement, OAP et servitudes) et applicables à sa parcelle. Dans cette perspective, il est prévu de faciliter l'accès à la réglementation par des applications numériques permettant d'avoir en ligne l'équivalent d'un certificat d'urbanisme à partir de la simple adresse ou de la référence cadastrale. Quelques grandes villes le proposent. Le plus souvent il faut se référer à des PDF, voire à des documents en papier disponibles en mairie. La réforme, en cours, de dématérialisation des planifications et des servitudes ${ }^{4}$ devrait régler d'ici 2020 ce problème persistant en systématisant le versement des documents sur le géoportail de l'urbanisme.

Figure 2. Rappel des documents présents dans un PLU (ou un PLUi)

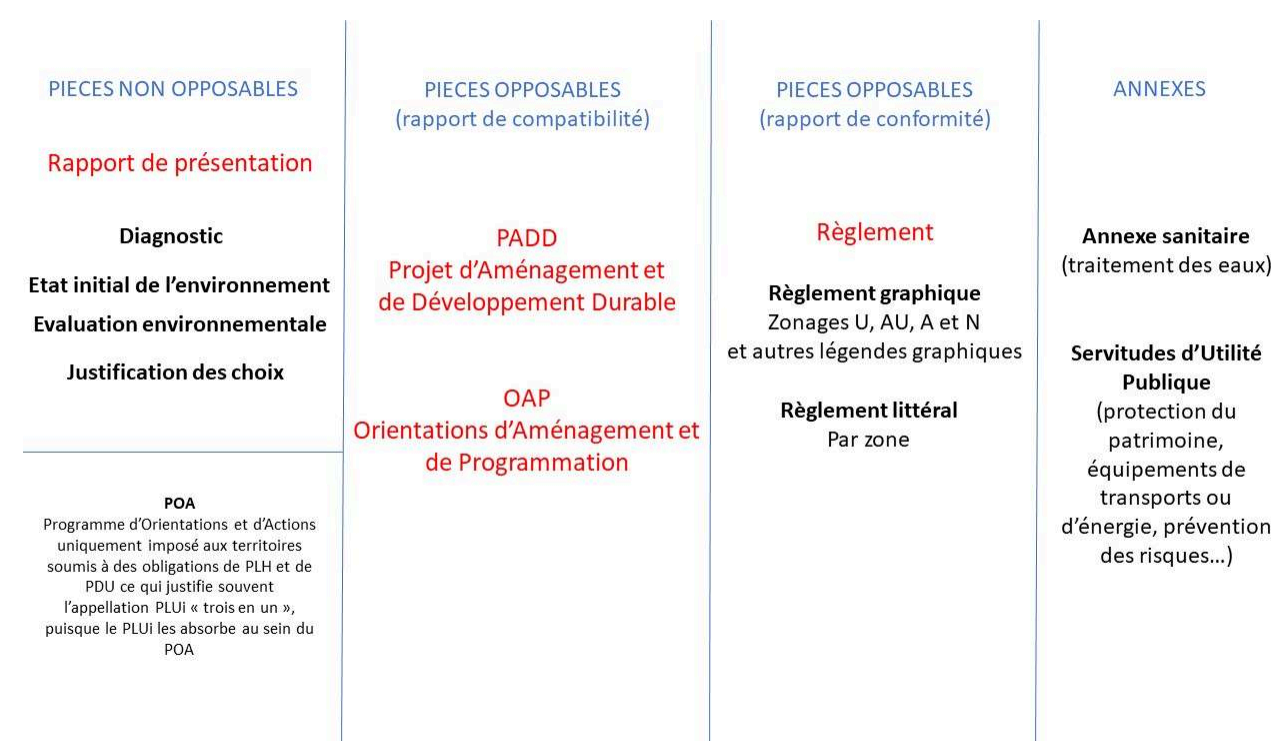

(c) Arnaud de Lajartre et Mathieu Gigot, 2018.

11 Mais la question de l'accessibilité aux règles du PLU ne se pose pas que sur un plan matériel : une fois entre les mains, encore faut-il les comprendre, ce qui n'est pas chose aisée en raison de la technicité de nombre d'entre elles (règles d'implantation et de 
recul, hauteurs, stationnement, etc.). Mais certaines règles, a priori moins techniques, n'en restent pas pour autant aisément lisibles; il s'agit de toutes celles se rapportant à la "qualité urbaine, architecturale, environnementale et paysagère » du règlement, dont le code qui retient cette expression n'apporte pas pour autant la définition. Cet enjeu paysager global se traduit en particulier au travers de la mise en œuvre dans le PLU des articles L. 151-17 à L. 151-25 du code de l'urbanisme (dédiés à la « qualité du cadre de vie »). L'article du règlement des PLU le plus directement relié à cette exigence urbanistique est l'article relatif à l'aspect extérieur des constructions (anciennement l'article 11 dans les règlements de POS ou de PLU avant la recodification de 2015). Quelle que soit la précision de cet article dans le règlement de PLU, le pétitionnaire se trouve nécessairement confronté à la question de l'intégration paysagère de son projet de construction, car cette exigence résulte également du code dont l'article R. 111-275 impose que «le projet [puisse] être refusé ou n'être accepté que sous réserve de l'observation de prescriptions spéciales si les constructions, par leur situation, leur architecture, leurs dimensions ou l'aspect extérieur des bâtiments ou ouvrages à édifier ou à modifier, sont de nature à porter atteinte au caractère ou à l'intérêt des lieux avoisinants, aux sites, aux paysages naturels ou urbains ainsi qu'à la conservation des perspectives monumentales ». Ainsi, qu'il s'agisse d'un article du PLU ou de l'article R. 111-27, le demandeur d'un permis de construire, ou d'une simple déclaration de travaux, doit comprendre les enjeux paysagers dans lesquels son projet s'intègre. Or, rien n'est moins simple en matière de paysage, car si le sujet appartient à tout le monde, chacun le comprend à sa façon. Ou ne le comprend pas, lorsqu'il s'agit de le relier à son propre projet... Le remplacement de persiennes en bois par un volet PVC roulant extérieur, la pose d'un portail métallique de 2 mètres là où n'existait avant aucune fermeture sur la rue, l'arrachage de haies anciennes dans un milieu bocager périurbain, le choix d'une mauvaise couleur de bardage métallique dans une zone d'activités, etc.: autant de sujets sur lesquels le pétitionnaire ne saisit pas toujours d'emblée les impacts sur le paysage.

Il est alors nécessaire de faire preuve de pédagogie auprès des demandeurs afin de les doter, le plus souvent, de deux clés de lecture de leur environnement conformément à la définition de la CEP. Tout d'abord le paysage ne se résume pas au "grand paysage " ou au "beau paysage ", celui des cartes postales du territoire, celui qui justement déclenche l'association d'idées permettant aussi, pendant la concertation préalable, de parler d'un sujet connu. Cette pédagogie du paysage sensibilise alors le demandeur à l'idée que tous les paysages se valent, y compris ceux dits ordinaires et de proximité, voire même les paysages souvent considérés comme dégradés, ceux des zones d'activités ou des entrées de ville, souvent du périurbain. Par ailleurs, après avoir informé/formé le pétitionnaire sur cette acception du paysage, il est nécessaire de l'éduquer sur l'impact que lui-même peut avoir sur son paysage par ses propres travaux, en lui démontrant comment, progressivement, de simples changements d'huisseries ou des extensions mal positionnées transforment, voire altèrent un paysage urbain local.

Mais pour réveiller cette conscience paysagère chez l'habitant d'un quartier, chez le promoteur immobilier ou le chef d'entreprise d'une zone d'activités, encore faut-il pouvoir mettre en face de son projet une personne-ressource. Dans certains territoires, comme des villes d'importance ou au contraire des communes rurales, des architectesconseils, ayant souvent participé à la fabrique du PLU, reçoivent des habitants afin de 
les aider à concevoir leurs projets de travaux, assumant à cette occasion une médiation de la réglementation d'urbanisme, en particulier sur les enjeux d'intégration paysagère. Tel est le cas de nombreux PNR ou bien entendu des CAUE, lorsque les budgets en crise des départements n'ont pas saigné leurs effectifs... Certains territoires aux enjeux paysagers majeurs n'ont pas hésité à recruter volontairement un architecteconseil, comme l'a fait par exemple la vallée de la Vézère afin d'épauler les agriculteurs dans le cadre de leurs projets de bâtiments au sein du site classé, par ailleurs inscrit au patrimoine mondial. Le service instructeur de la commune ou de l'intercommunalité peut bien entendu jouer ce rôle lorsque sa charge de travail l'y autorise, et qu'il s'estime lui-même apte à faire de la pédagogie citoyenne sur le thème du paysage.

En effet, la formation des instructeurs ne les dote pas toujours des clés de compréhension des enjeux paysagers tels que retenus par les documents réglementaires du PLU. Souvent géographes ou juristes de formation, les instructeurs des ADS (autorisations du droit des sols) peuvent aussi occuper leurs postes après un parcours dans la fonction publique territoriale très éloigné de l'urbanisme, et en particulier $\mathrm{du}$ thème du paysage. Faute de formation en la matière, le sujet n'est alors pas évident à traiter lors de l'instruction de permis de construire ou d'aménager. À cela s'ajoutent la charge de travail et la cadence de traitement des dossiers, en particulier dans les villes, favorisant dans les services instructeurs une préférence pour certains types de règles des PLU: il est plus facile d'appliquer rapidement des critères techniques et parfois purement arithmétiques (métrage du recul pour l'implantation de la construction, hauteur à l'égout, nombre de places de stationnement) que des critères qualitatifs (la valeur pittoresque par exemple), ceux-là même qui sont d'ordinaire mobilisés en matière de paysage. Ce volet du PLU renvoie logiquement à l'appréciation de l'esthétique des travaux envisagés par le pétitionnaire, souvent résumée sous l'expression d'une "intégration harmonieuse" de la construction dans son environnement. Chacun conviendra qu'apprécier ce critère majeur pour le paysage urbain peut se révéler plus complexe et plus chronophage que de vérifier la hauteur déclarée du faitage. Cela nécessiterait pour les instructeurs en ADS une vraie formation en matière de paysage. Or, dans un contexte de réforme régulière des droits de l'urbanisme et des collectivités territoriales (depuis le début des années 2010, une loi par an a minima), la priorité est donnée par les élus à l'actualisation des connaissances des agents quant au régime juridique des ADS et à leurs documents de référence.

Une solution de substitution passe alors par la formation entre services de la collectivité, quand celle-ci bénéficie évidemment d'une certaine taille, et que parfois elle a élaboré elle-même son PLUi. Sans raisonner de manière globale et théorique sur le paysage, la collectivité peut tout simplement expliquer aux agents chargés de l'instruction des ADS dans quel sens interpréter telle ou telle disposition du règlement ou d'une OAP. Cette pédagogie du paysage interservices se développe souvent lors de l'instruction d'un dossier réel de demande de travaux, l'étude de cas devenant ainsi le meilleur support pédagogique pour donner du sens à ce paysage «abstrait » visé par le PLU. La proximité physique du service instructeur et du service ayant piloté l'élaboration du PLU facilite évidemment cette dynamique de formation interne à la collectivité $^{6}$; par exemple, à Angers Loire Métropole, un étage séparant les deux services au sein du même bâtiment, il est fréquent qu'un instructeur descende quelques marches pour interroger l'architecte du patrimoine ou le chargé de mission paysage afin de voir avec elle/lui comment répondre à la demande de travaux en intégrant la qualité paysagère urbaine ou rurale. Quand elle fonctionne ainsi, cette pédagogie du 
paysage ne s'arrête d'ailleurs pas là : les retours ainsi produits du service instructeur sur l'application du PLU alimentent la réflexion sur les évolutions futures du document. Ces échanges avec le service instructeur existent d'ailleurs de plus en plus souvent et ce même lorsque la rédaction du PLU est sous-traitée à un bureau d'études ou à un cabinet d'urbanistes.

Le PLU offre ainsi potentiellement plusieurs temps de pédagogie paysagère. Néanmoins l'analyse des PLU retenus dans notre programme de recherche révèle que, sur le fond, ils sont susceptibles de former les citoyens au paysage d'une manière particulière : une lecture fine du contenu des PLU met en lumière un glissement sémantique du mot "paysage ", pouvant alors contribuer à nourrir des représentations citoyennes partielles de cet objet.

\section{Une pédagogie du paysage marquée par un glissement sémantique : du paysage au patrimoine}

Le paysage est fortement mobilisé dans les PLU, mais le sens qui lui est attribué varie souvent d'une pièce à l'autre comme s'il n'était que partiellement saisissable par le droit. En même temps, il reste une composante essentielle des documents non opposables qui accompagnent les documents d'urbanisme.

\section{Le paysage dans les différentes pièces du PLU : une notion mouvante réinterprétée}

18 Nous avons vu que la notion de paysage était abondamment appelée dans les documents de planification urbaine, parce qu'elle suggère à la fois l'idée d'une évolution de la ville, de ses formes et de son environnement, mais aussi celle du cadre de vie des habitants. Son acception oscille néanmoins selon les pièces qui composent le PLU entre deux tendances assez marquées que nous allons développer.

19 Pris au sens large dans le rapport de présentation, le paysage désigne à la fois ce qui relève du bâti et du non-bâti, c'est-à-dire autant les pleins que les vides et leurs interactions. Cette définition a souvent conduit les concepteurs de PLU à parler d'ambiances et, dans cette acception, le paysage est conforme à la définition établie dans la CEP. Si pour beaucoup, le PLU est avant tout un document d'urbanisme visant à réglementer l'occupation du sol et les formes urbaines, il constitue aussi l'expression d'une volonté politique locale mais qui reste vague, sinon commune, en particulier dans le Projet d'aménagement et de développement durable (PADD). L'analyse des PLU de notre échantillon montre que le paysage demeure très présent dans les textes car il permet de rendre compte des évolutions du territoire mais il est aussi le support d'un projet politique dont les habitants sont invités à se saisir. Nombreux sont les PLU qui, dans leur PADD, prennent appui sur le paysage pour formuler des orientations politiques quant à l'évolution du territoire. À cet égard, les termes de "préservation ", "protection» ou "valorisation" sont fréquemment mobilisés pour caractériser le paysage dans le projet.

En fonction des pièces du PLU, le paysage est caractérisé différemment, comme si ce qu'il recouvrait se précisait en fonction de la nature - opposable ou non - des parties du document d'urbanisme. On repère un effet entonnoir conduisant à préciser - et 
restreindre - ce que signifie le paysage. Au fil de la lecture des différentes pièces du PLU, la notion de paysage s'amenuise, partant d'une acception assez large dans les documents non opposables avant d'être souvent réduite à sa composante naturelle, voire en tant que patrimoine à préserver dans les documents opposables. L'effet entonnoir provoque un éclatement de la notion de paysage en deux acceptions distinctes, qui prennent un tout autre sens (figure 3), et qui ont une incidence sur ce que les habitants comprendront du paysage. Les rédacteurs des PLU distinguent donc, dans les pièces réglementaires du PLU, le paysage naturel, c'est-à-dire non bâti, du paysage bâti qu'ils qualifient souvent de patrimoine. Autrement dit, la notion de paysage perd de sa substance et devient in fine une ressource à protéger plus qu'un état dont l'évolution pourrait être gérée et accompagnée.

Figure 3. La variation du sens du paysage dans les pièces du PLU

\begin{tabular}{|c|c|c|c|c|c|}
\hline \multicolumn{4}{|c|}{ PIECES NON OPPOSABLES } & & PIECES OPPOSABLES \\
\hline Rapport de présentation & 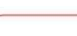 & PADD & 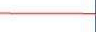 & & Règlement \\
\hline $\begin{array}{l}\text { PAYSAGE = } \\
\text { Bâti et non bâti, } \\
\text { cadre de vie, } \\
\text { ambiances... }\end{array}$ & & $\begin{array}{l}\text { PAYSAGE = } \\
\text { Bâti et non bâti, } \\
\text { cadre de vie, } \\
\text { ambiances... }\end{array}$ & & & $\begin{array}{l}\text { PAYSAGE = } \\
\text { OU éléments bâtis } \\
\text { OU éléments naturels } \\
\text { pas d'interaction }\end{array}$ \\
\hline $\begin{array}{l}\text { Etat initial de l'environnement } \\
\text { descriptif, sans forme juridiquement } \\
\text { contraignante }\end{array}$ & Justifie & $\begin{array}{l}\text { Projet politique qui } \\
\text { définit les grandes } \\
\text { orientations }\end{array}$ & $\frac{\text { Trouve }}{\text { traduction }}$ & sa & $\begin{array}{l}\text { Zonage et } \\
\text { règlement littéral } \\
\text { qui encadre l'affectation } \\
\text { et le droit des sols }\end{array}$ \\
\hline
\end{tabular}

(c) Mathieu Gigot, 2018.

21 On pourrait voir dans ce processus une forme d'incapacité du droit à se saisir d'une notion large, englobant les éléments bâtis et non bâtis, tout autant que la perception que peuvent en avoir les usagers. Dans le même temps, les PLU ne peuvent s'abstraire de convoquer le paysage : les documents d'urbanisme doivent l'intégrer explicitement depuis $1993^{7}$, ambition confirmée depuis la loi ALUR de 2014 qui traduit en droit positif les ambitions de la CEP. Si la préservation des paysages (notamment considérés comme exceptionnels) est encouragée par l'État, la gestion des paysages plus quotidiens est aussi un enjeu majeur. En la matière, si les PLU peuvent avancer dans les pièces non opposables le principe d'une gestion équilibrée des paysages par des aménagements raisonnés, ils se concentrent dans les pièces opposables majoritairement sur les conditions de la protection d'une ressource qualifiée de patrimoniale (ou considérée comme telle). L'idée de paysage ainsi renvoyée aux habitants est tronquée puisque l'attention se porte sur des éléments remarquables (ou remarqués) qu'il convient de protéger sans considération pour les paysages aux qualités plus ordinaires. À l'intérieur même des PLU, on constate cette évolution de l'acception du paysage qui, au départ, embrasse toutes les facettes de la notion, en particulier dans les documents non opposables du PLU (rapport de présentation et PADD) pour finalement se cantonner à la seule protection de sa composante végétale dans le règlement. Si l'habitant ne lit pas l'ensemble des pièces du PLU et s'il ne réalise pas de lien entre ces différentes pièces, il ne retiendra que la définition réductrice du paysage véhiculée par les parties réglementaires du PLU, c'est-à-dire celles qui encadrent et définissent les contours de 
son projet. Ainsi, il est possible que les citoyens n'assimilent qu'une définition partielle, végétale ou patrimoniale, du paysage.

L'analyse de plusieurs PLU menée dans le projet de recherche montre bien cet écart entre l'identification des paysages dans les documents non opposables et l'appauvrissement du sens qui leur est donné au sein du règlement. À Orléans ${ }^{8}$, le PLU reprend la définition de la CEP dans un chapitre dédié à l'analyse paysagère: « agencement des éléments matériels qui composent les lieux, vécu d'une société sur le territoire qu'elle modèle, et regard que chacun peut porter sur l'espace ${ }^{9}$ ». Malgré tout, la notion de paysage glisse vite vers celle d'ambiance, plus large et moins tangible. L'ambiance est une notion difficile à circonscrire: "elle est plus large que celle du cadre de vie, qu'elle englobe en y intégrant les représentations, individuelles et collectives, du plaisant, du confortable, du salubre » (Pumain, Paquot, Kleinschmager, 2006, p. 13). Cette notion est massivement mobilisée dans le rapport de présentation du PLU d'Orléans, qui l'utilise comme une façon d'agréger les éléments du cadre bâti avec ceux plus «naturels ». Autrement dit, les concepteurs du PLU cherchent à définir des paysages vécus, intériorisés et qui participent à la définition du cadre de vie. Une souspartie est consacrée à ces «ambiances urbaines " qui «s'échelonnent d'une urbanité intense dans les espaces les plus centraux à une atmosphère presque rurale dans certains espaces de périphérie ${ }^{10} »$. Ici le paysage est bien considéré comme le rapport entre le bâti et le non-bâti et ses interrelations : le PLU cite « une palette de lieux aux atmosphères différentes notamment du fait des rapports de composition entre le bâti, l'espace public et la végétation ${ }^{11} »$ (figure 4). Schématisée par le dessin, elle est plus facilement perceptible par le lecteur.

Figure 4. Exemple de qualification des ambiances urbaines dans le PLU d'Orléans
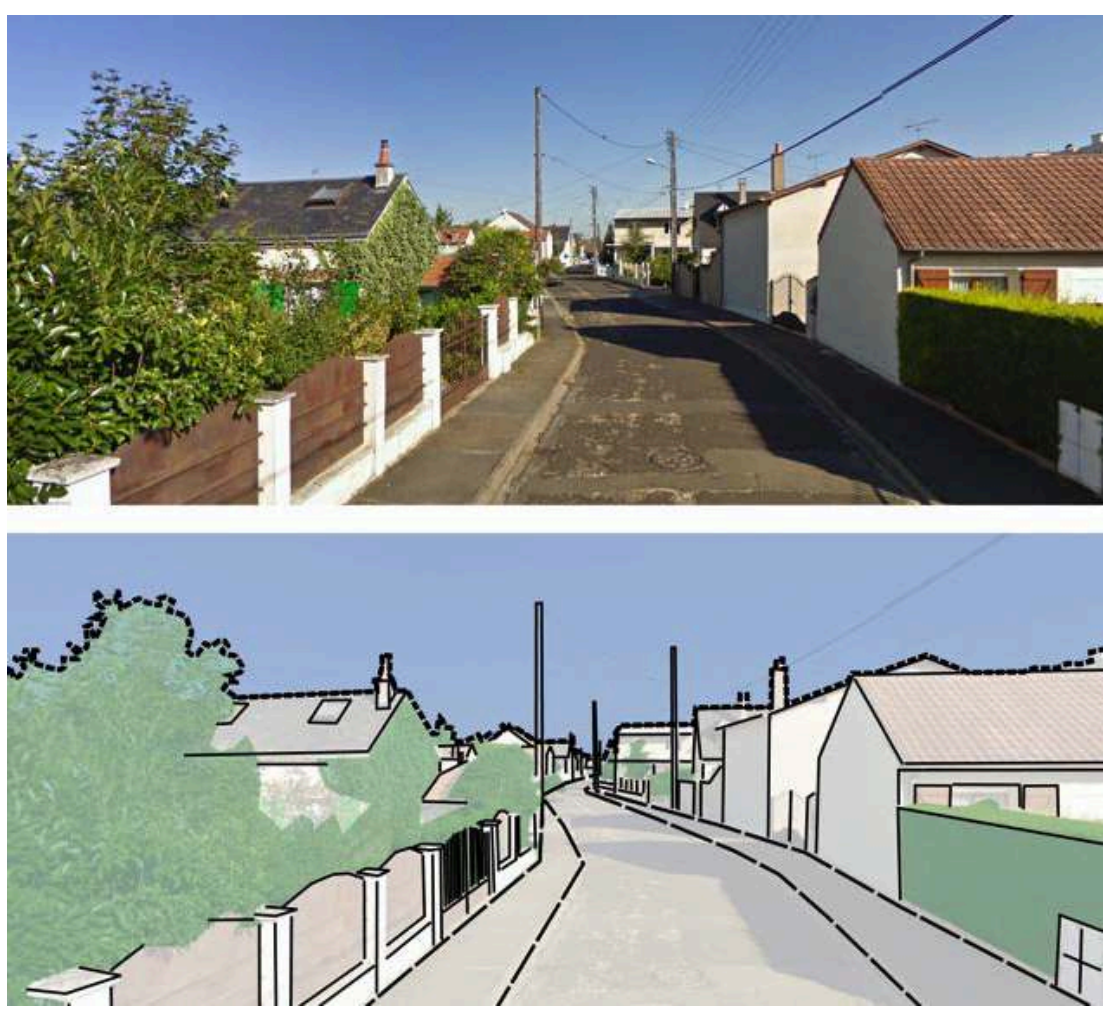

Ambiance urbaine, rue Jules Noël.

(c) Extrait du PLU d'Orléans, 2013 
Certains PLU excluent d'emblée la notion d'un paysage habité pour ne retenir que celle d'un paysage naturel. C'est le cas du PLU de Montoire-sur-le-Loir ${ }^{12}$ où le rapport de présentation décrit le «contexte paysager ${ }^{13}$ » en ne s'appuyant que sur les caractéristiques morphologiques du paysage, afin de définir des unités paysagères exemptes de tout habitat comme le montre la figure 5 qui présente la carte de l'analyse paysagère de la commune. Seuls les monuments historiques sont appelés à apparaître sur la carte, ce qui exprime bien, en creux, la dimension patrimoniale donnée au paysage.

Figure 5. L'analyse paysagère à Montoire-sur-le-Loir, centrée sur les composantes naturelles du paysage

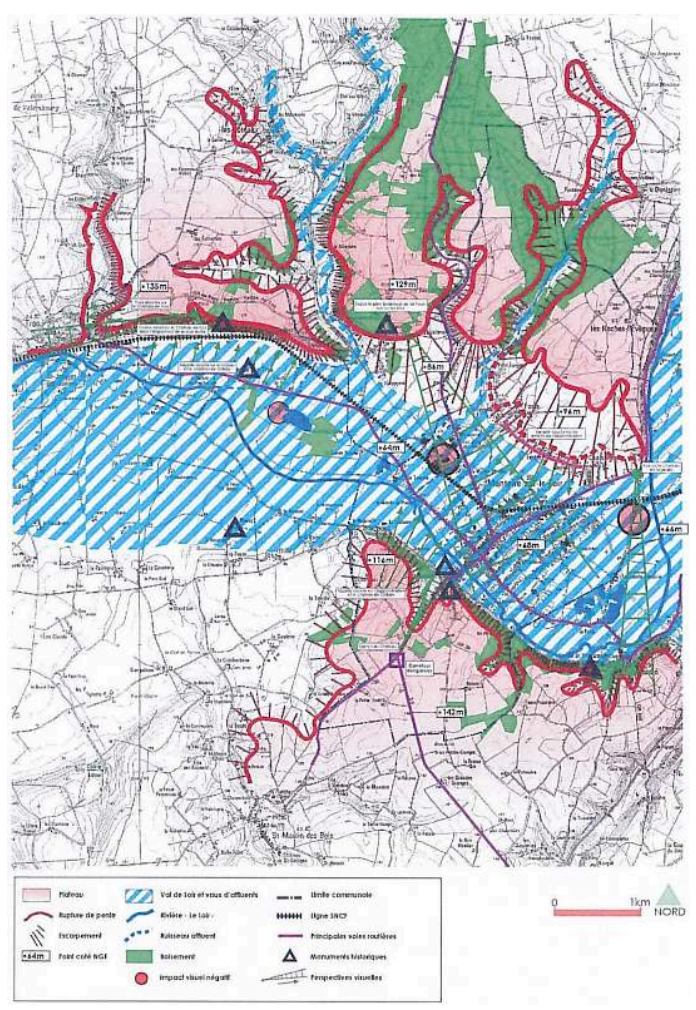

(c) Extrait du PLU de Montoire-sur-le-Loir, 2006.

Dans les choix retenus pour le PADD, le paysage devient patrimoine naturel: «Le paysage montoirien réside dans l'harmonie entre les différents éléments qui le constituent : le Val de Loir et ses vallons, les coteaux, les boisements ${ }^{14}$. Les éléments du paysage bâti, eux, sont fondus dans un chapitre consacré aux «formes urbaines, architecturales et du patrimoine ». Plus loin, les justifications du règlement distinguent elles aussi ce qui relève de "la protection du paysage » et de "la protection du patrimoine architectural et historique ${ }^{15} »$.

De façon plus discrète, le même changement s'opère au fil de la lecture du PLU d'Orléans. La notion d'ambiance qui a permis de définir la palette des paysages orléanais s'étiole. Dans le PADD, un premier glissement s'opère : après avoir parlé du paysage, le rédacteur évoque plutôt le patrimoine. Les paysages précédemment décrits sont dilués dans un item plus large: "préserver et mettre en valeur le patrimoine remarquable ${ }^{16} »$. Ainsi, le paysage devient une ressource à protéger, un bien patrimonial. 
26 L'idée d'un paysage patrimonialisé revient d'ailleurs souvent dans les pièces réglementaires des PLU; le paysage y est réduit à ses composantes naturelles, considérées comme des biens du "patrimoine vert». À Orléans, par exemple, les dispositions générales du règlement précisent la nature des éléments protégés au titre du patrimoine et des paysages : « cœurs de jardin, parcs, alignements d'arbres protégés, et arbres isolés ${ }^{17}$ ». Le citoyen qui lira le règlement retiendra que paysage et patrimoine se rejoignent et que les ambiances urbaines décrites précédemment ont vocation à être préservées. Cette dimension est très marquée à Rennes ${ }^{18}$, où c'est le paysage en tant que composante patrimoniale qui est pris en compte dès les premières pièces du PLU. Le rapport de présentation consacre d'ailleurs l'expression de «patrimoine vert » dans l'un de ses sous-titres ${ }^{19}$. Ce dernier est mis en rapport avec le patrimoine bâti, au même niveau, en ce que les deux constituent « un composant essentiel dans la structure et l'organisation de la ville ${ }^{20} »$. Ici, le rédacteur sépare d'emblée ce qui relève du paysage bâti et du paysage naturel mais avec une acception patrimoniale affirmée pour ces deux composantes paysagères: dans les deux cas, il donne une valeur patrimoniale au paysage avec un enjeu de préservation. Comme à Orléans, le paysage est ici un élément fondamental du cadre de vie des habitants qu'il convient de protéger. Plus encore, le rapport de présentation du PLU de Rennes définit les fondements de ce que les rédacteurs nomment "les caractéristiques de l'image paysagère de Rennes ", c'est-àdire «les éléments qui contribuent à donner une image spécifique de la ville de Rennes ${ }^{21}$ » et qui, donc, acquièrent une certaine valeur patrimoniale. Le paysage est alors considéré dans toute sa diversité même si l'on constate une séparation entre ce qui relève des composantes bâties d'une part et végétales d'autre part. Ainsi, le PADD du PLU de Rennes reprend cette différenciation et ne parle plus de paysage : dans une première orientation, le PADD prend en compte le patrimoine bâti ${ }^{22}$ puis dans une autre le patrimoine vert ${ }^{23}$, avec l'ambition de protéger ces deux types de patrimoines. Cependant, le PLU de Rennes va plus loin - réglementairement - que celui d'Orléans car il mobilise dans le règlement graphique un zonage $\mathrm{NE}^{24}$, qui correspond aux zones naturelles protégées, et un zonage UP ${ }^{25}$, qui correspond aux secteurs patrimoniaux urbains. Ces zonages spécifiques sont formalisés comme tous les autres, sans intention pédagogique particulière. Ce sont d'autres outils, non réglementaires, qui feront ce travail de médiation. En tout état de cause, ces différents zonages montrent que le paysage est découpé en deux parties distinctes : ce qui relève du patrimoine bâti, d'une part, et ce qui relève du patrimoine naturel, d'autre part. La même distinction s'observe dans beaucoup de règlements écrits : à Tours ${ }^{26}$ par exemple, les règles relatives au patrimoine bâti sont contenues dans l'article 11 du règlement (« Aspect extérieur des constructions " : façades, toitures, murs de clôtures, etc.) alors que les règles relatives au végétal sont réunies dans l'article 13 (« Espaces libres, plantations et espaces boisés classés » : arbres, haies, plantations à réaliser, etc. ${ }^{27}$. La notion de paysage est donc scindée en plusieurs acceptions selon les articles du règlement.

L'approche du paysage par le règlement d'urbanisme conduit à des prescriptions s'attachant à des objets tangibles. Cette matérialité exacerbée du paysage explique en partie le glissement sémantique que nous évoquions : le droit semble ici incapable de saisir le paysage dans toute sa complexité et de traduire, dans un règlement, les questions de perception ni même d'ambiance. Cela passe forcément par un support matériel bâti ou non bâti. C'est aussi pour cette raison que le paysage devient patrimoine, dans la mesure où les prescriptions s'attachent à protéger les abords du bâti existant, notamment lorsqu'il s'agit des notions d'insertions paysagères. 


\section{Une pédagogie du paysage à travers les documents d'accompagnement}

28 En plus du PLU, d'autres documents permettent une appropriation du paysage par les habitants. Parmi eux, certains ne sont pas opposables et constituent des appuis non négligeables à l'établissement d'une pédagogie du paysage. Ils sont d'ailleurs mobilisés lors de l'élaboration du PLU ou peuvent même servir aux collectivités pour appuyer leurs arguments lors de l'instruction des autorisations d'urbanisme, afin de mieux expliquer aux pétitionnaires les enjeux paysagers d'un projet. Ces documents sont tous à la fois des outils de compréhension pour les citoyens et des outils d'aide à la décision pour les élus, en particulier lorsque ces outils n'ont pas d'effet juridique réel. Deux catégories de documents peuvent être distinguées : les documents de connaissance du paysage et les plans d'action.

La connaissance et la caractérisation des paysages sont des préalables essentiels à l'action. La généralisation des atlas du paysage, dont la dimension pédagogique est affichée par les services déconcentrés de l'État qui les réalisent, permet de suivre les transformations des paysages et de mieux encadrer leur évolution, notamment au travers des orientations de qualité paysagère reprises et développées par les acteurs en charge de l'élaboration des documents d'urbanisme. Des plans d'action sont ainsi définis sans nécessairement avoir de dimension prescriptive. Il en va ainsi pour les plans de paysages, démarche initiée et soutenue par l'État qui vise à faciliter la compréhension et la prise en compte des paysages dans les différents documents d'urbanisme. Ces démarches sont aussi l'occasion de mener des diagnostics paysagers qui mobilisent les acteurs des territoires, qu'ils soient experts, décideurs ou usagers.

Les notions sur le paysage sont en effet transmises aux habitants par ces documents complémentaires aux outils réglementaires. En particulier lorsqu'il s'agit de documents d'urbanisme patrimoniaux qui prennent par ailleurs en compte les paysages dans les pièces opposables, les collectivités locales tentent de mettre en œuvre des outils annexes. Qu'ils adoptent la forme de chartes, de cahiers de bonnes pratiques ou de plaquettes de sensibilisation, ces outils relèvent de ce qu'il est désormais convenu de nommer le «droit souple ${ }^{28} »$. Certes non opposables, ils sont néanmoins parfois convoqués lors des débats autour de l'insertion paysagère des projets car ils permettent de contextualiser le projet dans son environnement. La palette d'outils ainsi mobilisée va du «non-droit » au «droit dur » (figure 6), saisissant différemment la notion de paysage et donc orientant le sens qu'il lui est accordé. 
Figure 6. Une palette d'outils du « non-droit » au « droit dur »

\begin{tabular}{|c|c|c|c|}
\hline NON DROIT & & DROIT SOUPLE & DROIT DUR \\
\hline $\begin{array}{l}\text { Plaquettes d'information } \\
\text { ou de médiation }\end{array}$ & $\begin{array}{l}\text { Liste des essences } \\
\text { D'arbres à privilégier }\end{array}$ & $\begin{array}{c}\text { Chartes } \\
\text { (paysagères, } \\
\text { Architecturales, PNR...) }\end{array}$ & $\begin{array}{l}\text { Prescriptions réglementaires } \\
\text { Nuancier intégré au règlement }\end{array}$ \\
\hline $\begin{array}{l}\text { Inventaires en ligne nationaux } \\
\text { (Mérimée, ZNIEFF) } \\
\text { et locaux (par ville) }\end{array}$ & $\begin{array}{l}\text { Cahiers de bonnes } \\
\text { pratiques }\end{array}$ & $\begin{array}{c}\text { Plans d'actions } \\
\text { (plans de paysage...) }\end{array}$ & $\begin{array}{l}\text { Inventaire annexé au règlement } \\
\text { (avec fiches prescriptives) }\end{array}$ \\
\hline $\begin{array}{l}\text { Nuancier indicatif } \\
\text { en annexe du PLU }\end{array}$ & $\begin{array}{l}\text { Recommandations } \\
\text { insérées dans le } \\
\text { règlement }\end{array}$ & $\begin{array}{l}\text { Orientatic } \\
\text { Et de }\end{array}$ & \\
\hline
\end{tabular}

Non contraints par des normes d'écriture ou de présentation spécifiques, les documents d'accompagnement sont très illustrés et permettent de rendre compte de la complexité des thématiques qu'ils traitent. Sur la question du paysage, en particulier, ils peuvent comporter des photographies, des cartes ou encore des coupes qui montrent d'une façon simplifiée la consistance paysagère d'un territoire. Sur la Vallée de la Loire, inscrite au patrimoine mondial de l'Unesco, la mission Val de Loire, en charge de la gestion du site inscrit, a conçu et développé des blocs-diagrammes qui caractérisent les composantes territoriales de la valeur universelle exceptionnelle (VUE) (figure 7).

\section{Figure 7. Bloc-diagramme caractérisant la VUE à Amboise}

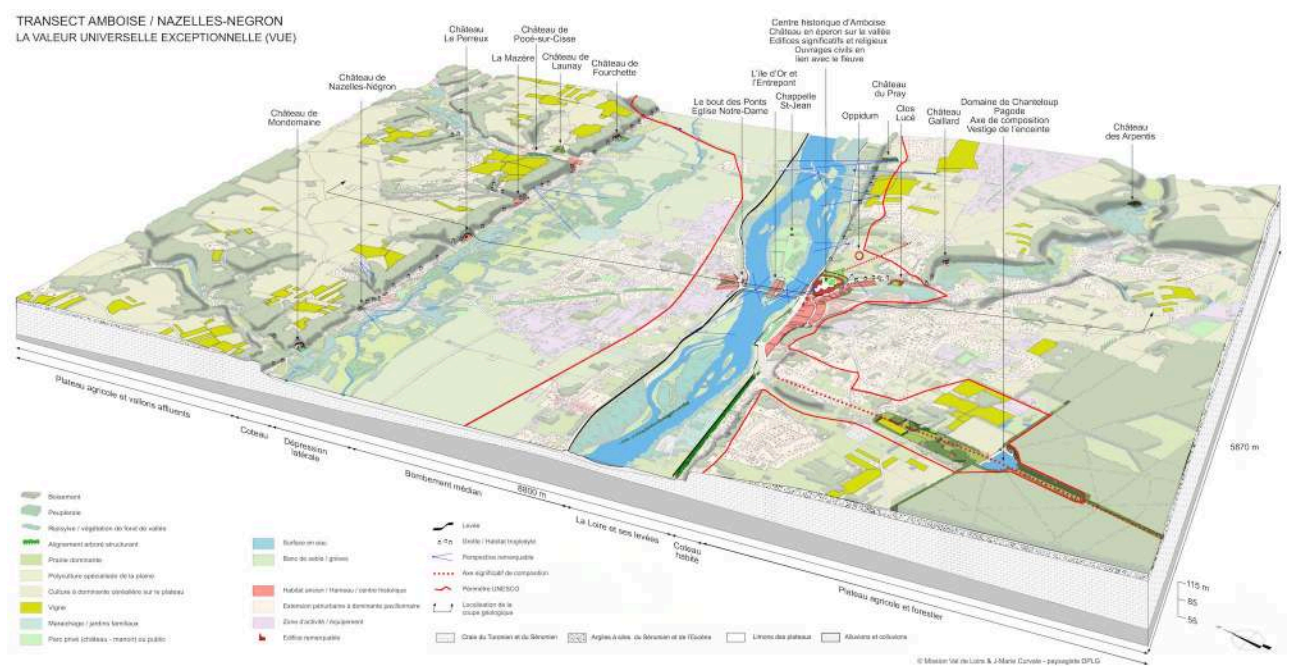

Extrait du Guide pratique Val de Loire patrimoine mondial et aménagement du territoire, 2017.

Sources : Mission Val de Loire patrimoine mondial/Jean-Marie Curval, paysagiste.

Cet outil permet de mieux appréhender le paysage et ce à plusieurs échelles. Selon les unités paysagères retenues, c'est le grand paysage qui peut être expliqué tout comme un paysage plus restreint qui parlera sans doute davantage aux habitants connaissant 
leur cadre de vie proche. C'est le parti pris par les acteurs de l'Aire de mise en valeur de l'architecture et du patrimoine (AVAP) ${ }^{29} \mathrm{~d}^{\prime}$ Amboise qui ont décliné des blocsdiagrammes à l'échelle de parcelles types pour aider les pétitionnaires à mieux appréhender leur territoire afin de comprendre les règles s'imposant à leur parcelle (figure 8).

Figure 8. Bloc-diagramme d'un espace type à Amboise

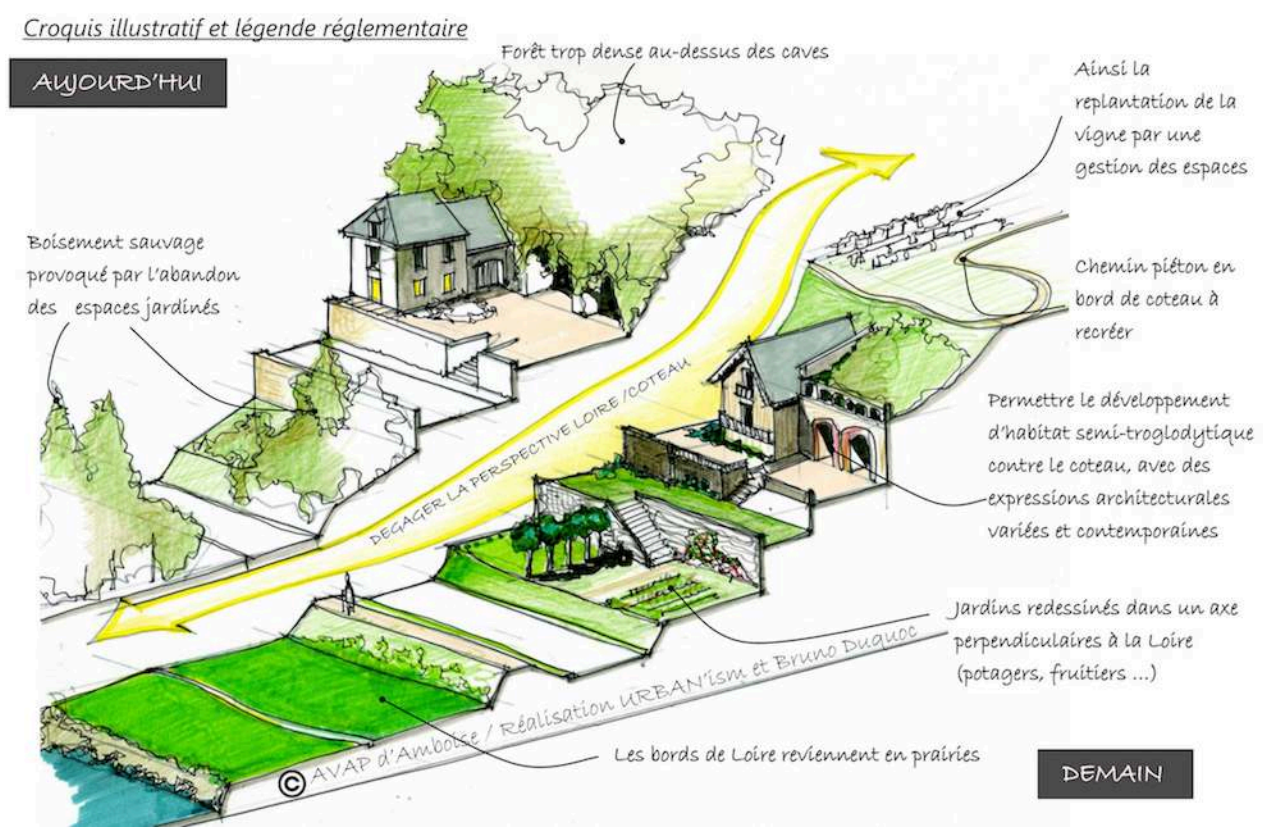

Extrait de l'AVAP d'Amboise, 2017.

Sources : URBAN'ism/Bruno Duquoc.

À partir de plusieurs catégories de paysages qui caractérisent la commune, le document donne à voir l'état initial du paysage et les ambitions qui lui sont attachées sur un même croquis. Cet «avant-après » est très parlant: les habitants peuvent d'un seul coup d'œil saisir les enjeux d'évolution des paysages qui les entourent.

Dans le même esprit, la charte architecturale et paysagère de la Vallée du Loir identifie par exemple des orientations générales qui, en se déclinant en 21 fiches action, permettent de sensibiliser les habitants au paysage dans toutes ses composantes. La forme de rédaction non contrainte a permis de rendre compte de la complexité des unités paysagères, notamment au travers de coupes schématiques qui permettent de saisir les enjeux paysagers (figure 9). 
Figure 9. Exemple de coupe schématique caractérisant une séquence paysagère dans la Vallée du Loir

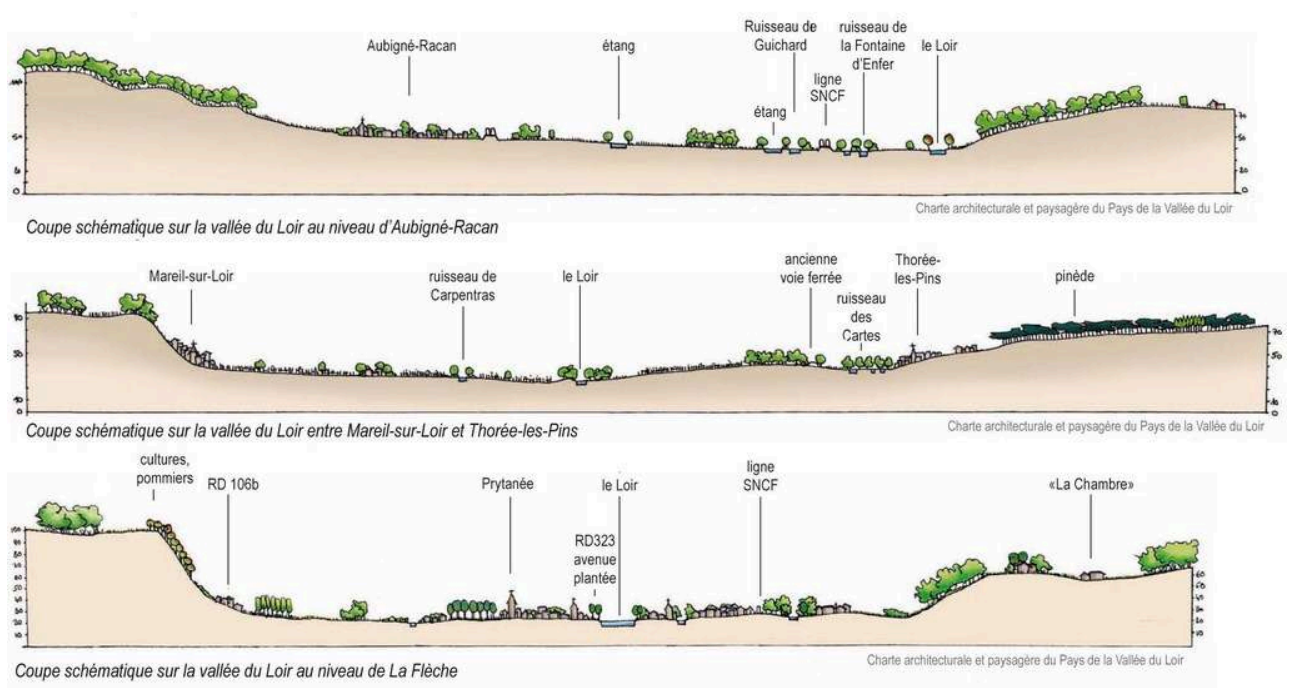

Extrait de la charte architecturale et paysagère du Pays de la Vallée du Loir, 2013.

La charte se décompose ainsi en plusieurs axes qui prennent à la fois en compte les formes bâties («valoriser les formes urbaines traditionnelles»; «concevoir des extensions urbaines en accord avec l'identité du territoire et les structures urbaines existantes $»^{30}$ ) et les usages du paysage ( accompagner une agriculture participant positivement au cadre de vie $»^{31}$ ).

Ce type de document ne remplace donc pas le règlement d'urbanisme mais présente l'intérêt d'accompagner l'habitant dans la compréhension des prescriptions, notamment au regard des enjeux paysagers. L'un des objectifs est de mieux encadrer les autorisations d'urbanisme et de les sécuriser juridiquement puisque la charte, même si elle ne s'apparente pas à du "droit dur ", vient appuyer les décisions des élus en illustrant par exemple le principe de l'insertion paysagère. C'est une pédagogie du paysage pour le citoyen mais aussi pour les élus et les instructeurs des autorisations d'urbanisme.

En conclusion, si le «droit dur " semble incapable de saisir le paysage dans toutes ses composantes, les outils d'accompagnement permettent, eux, de retranscrire la complexité du paysage, de ses différentes acceptions et de toutes les dimensions qu'elles recouvrent. Lorsqu'ils sont bien diffusés par les collectivités locales, ces documents sont un appui précieux pour aider les habitants à comprendre ce qu'est le paysage afin de mieux en gérer l'évolution, notamment lors de l'instruction des autorisations d'urbanisme. La possibilité pour le PLU de créer cette situation sociale d'apprentissage se trouve alors facilitée par la diversité des supports juridiques - et métajuridiques - qui formalisent le projet de paysage de la collectivité. Malheureusement, l'observation des terrains et des documents retenus dans le programme de recherche PLU patrimonial révèle que cette pédagogie du paysage s'accompagne rarement d'une pédagogie de la règle... Si les structures paysagères sont expliquées aux habitants, la règle de droit qui traduit les enjeux paysagers dans un document d'urbanisme est plus difficile à leur transmettre. 


\section{BIBLIOGRAPHIE}

Baffert, P., PLU et PLUi, Montrouge, Éditions Législatives, 2017, 286 p.

Barrière, 0 ., « Le paysage façonné par le droit, entre la rationalité du droit positif et l'empirisme culturel juridique ", Vertigo. La revue électronique en sciences de l'environnement, hors-série 14, septembre 2012, URL : http://journals.openedition.org/vertigo/12482.

Blanc, N., Glatron, S., « Du paysage urbain dans les politiques nationales d'urbanisme et d'environnement ", L'Espace géographique, T. 34, 2005/1, p. 65-80, URL : https://www.cairn.info/ revue-espace-geographique-2005-1-page-65.htm.

Cans, C. et Cizel, O., Loi biodiversité : ce qui change en pratique, Montrouge, Éditions Législatives, 2017.

Conseil d'État, « Le droit souple », octobre 2013, 297 p. URL : http:// www.ladocumentationfrancaise.fr/var/storage/rapports-publics/144000280.pdf.

Davodeau, H., Toublanc, M., « Le paysage outil, les outils du paysage - Principes et méthodes de la médiation paysagère ", « Outils pour décider ensemble - Aide à la décision et gouvernance ", colloque des 25-26 octobre à Montpellier, 2010, p. 375-391/436.

Hercé, S., Le PLU(i) : régime juridique, élaboration, évolution, contentieux, Paris, Le Moniteur, 2017, $398 \mathrm{p}$.

Labat, D., Donadieu, P., « Le paysage, levier d'action dans la planification territoriale ", L'Espace géographique, t. 42, 2013/1, p. 44-60, URL : https://www.cairn.info/revue-espacegeographique-2013-1-p-44.html.

Ministère du Logement et de l'Habitat durable, « Guide de modernisation du contenu du plan local d'urbanisme », avril 2017, 181 p. URL : http://www.cohesion-territoires.gouv.fr/IMG/pdf/ guide_de_la_modernisation_du_contenu_du_plan_local_d_urbanisme_-_avril_2017.pdf.

Noguellou, R., « La règle d'urbanisme et les PLU : où se trouve la règle d'urbanisme? », Revue française de droit administratif (RFDA), 2016, p. 872.

Pumain, D., Paquot, T., Kleinschmager, R. (dir.), Dictionnaire de la ville et de l'urbain, Paris, Économica, 2006, 320 p.

Sgard, A., « Le paysage dans l'action publique : du patrimoine au bien commun », Développement durable et territoires, vol. $1, \mathrm{n}^{\circ} 2$, septembre 2010, URL : http://developpementdurable.revues.org/ 8565.

Sgard, A., Rudaz, G., Géo-Regards. Les Dimensions politiques du paysage, n 8, Neuchâtel, Éditions Alphil, 2015.

Sgard, A., Fortin, M.-J., Peyrache-Gadeau, V., « Le paysage en politique », Développement durable et territoires, vol. 1, $\mathrm{n}^{\circ}$ 2, septembre 2010, URL : https://journals.openedition.org/ developpementdurable/8522.

Traoré, S., « Le plan local d'urbanisme intercommunal et la loi ALUR », Actualité juridique des collectivités territoriales (AJCT), 2014, p. 350-354.

Tribillon, J.-F., Le Droit nuit gravement à l'urbanisme, Paris, Éditions de la Villette, 2017.

Zask, J., « L'élève et le citoyen, d'après John Dewey », Le Télémaque, nº 20, 2001/2, p. 53-64, URL : https://www.cairn.info/revue-le-telemaque-2001-2-page-53.html. 


\section{NOTES}

1. Sur la base de ces liens, un «PLU patrimonial » est un PLU identifiant et protégeant à des degrés variables les patrimoines d'un territoire. Voir https://plupat.hypotheses.org.

2. Convention européenne du paysage adoptée le 20 octobre 2000.

3. http://www.club-plui.logement.gouv.fr/paysage-patrimoine-r116.html.

4. https://www.geoportail-urbanisme.gouv.fr.

5. Cet article important au sein du RNU (Règlement national d'urbanisme : la base réglementaire pour les communes sans planification urbaine) inspire les articles des PLU dédiés à la question de l'intégration paysagère des constructions, et peut même être appliqué par le juge en cas de contentieux si ce dernier estime les articles du PLU insuffisants sur le sujet.

6. La mutualisation des services engendrée par les regroupements intercommunaux peut d'ailleurs favoriser ce rapprochement entre service en charge de la planification urbaine et service instructeur désormais unique pour un certain nombre d'établissements publics de coopération intercommunale (EPCI) récemment créés. En échange de quoi l'habitant perd alors la proximité avec le service...

7. Loi $\mathrm{n}^{\circ}$ 93-24 du 8 janvier 1993 sur la protection et la mise en valeur des paysages (JORF 9 janvier 1993).

8. PLU d'Orléans (Loiret), approuvé le 25 octobre 2013.

9. PLU d'Orléans, rapport de présentation, t. 1, p. 126.

10. PLU d'Orléans, rapport de présentation, t. 1, p. 134.

11. Ibid.

12. PLU de Montoire-sur-le-Loir (Loir-et-Cher), approuvé le 11 avril 2006.

13. PLU de Montoire-sur-le-Loir, rapport de présentation, p. 38.

14. Ibid., p. 48.

15. Ibid., p. 55-56.

16. PLU d'Orléans, PADD, orientation 1, p. 22.

17. PLU d'Orléans, règlement, titre 1, p. 9.

18. PLU de Rennes (Ille-et-Vilaine), approuvé le 17 mai 2004.

19. PLU de Rennes, rapport de présentation, t. 1, p. 186.

20. PLU de Rennes, rapport de présentation, t. 1, p. 191.

21. PLU de Rennes, rapport de présentation, t. 1, p. 201.

22. PLU de Rennes, PADD, orientation 1 « Rennes, ville-centre d'une métropole régionale », p. 17.

23. PLU de Rennes, PADD, orientation 6 « Rennes, éco-ville», p. 51.

24. La zone NE du PLU de Rennes correspond à une zone naturelle de préservation et de mise en valeur du paysage. Elle recouvre également des secteurs présentant simplement un caractère naturel.

25. La zone UP du PLU de Rennes correspond à des zones urbaines dont la composition urbaine est homogène et qui constituent des ensembles patrimoniaux d'intérêt local.

26. PLU de Tours, approuvé le 18 juillet 2011.

27. PLU de Tours, règlement littéral.

28. Rapport annuel du Conseil d'État de 2013.

29. Depuis la loi LCAP du 7 juillet 2016, les AVAP sont transformées en sites patrimoniaux remarquables.

30. Charte architecturale et paysagère de la Vallée du Loir, axe 1.

31. Charte architecturale et paysagère de la Vallée du Loir, axe 2. 


\section{RÉSUMÉS}

En abordant de façon importante le paysage dans leurs différentes pièces, les PLU contribuent sur le terrain à une forme de pédagogie du paysage auprès des citoyens ; cela se produit à deux niveaux, qui feront l'objet de nos deux parties. Tout d'abord l'élaboration d'un PLU, puis sa mise en œuvre lors des demandes d'autorisation de travaux permettent de créer des situations sociales d'apprentissage autour du paysage, au travers des échanges entre collectivité et habitants, puis entre pétitionnaires et service instructeur; à ce moment, quel que soit le sens donné au mot paysage, les débats génèrent un temps didactique. Néanmoins, sur le fond, nous verrons dans un second temps que cette pédagogie du paysage repose souvent sur un glissement sémantique entre paysage et patrimoine, variant selon l'opposabilité des pièces du PLU. Du paysage cadre de vie dans le PADD, on passe souvent à un paysage purement patrimonial dans le règlement. Ce glissement favorise donc une représentation orientée du paysage pour les habitants, au service de laquelle s'inscrivent plusieurs documents de médiation.

Local urban development plans (in French, Plan local d'urbanisme, PLU) in their various components focus greatly on the landscape thus contributing in the field to a form of pedagogy of the landscape for citizens; this takes place at two levels which will be addressed in the two parts of the article. Social situations which teach about the landscape occur in the elaboration of the PLU and in its implementation with the submissions for building permits involving exchanges between the local authorities and the inhabitants and subsequently between petitioners and the building permit office. Whatever meaning is given to the term "landscape", these discussions provide didactic opportunities. However, we will demonstrate that in substance this pedagogy of the landscape is often founded on a semantic shift between landscape and heritage, which varies according to the opposability of the components of the PLU. From the landscape as a living environment in a sustainable urban development plan there is often a shift towards a regulatory framework defining the landscape purely in heritage terms. For the inhabitants this fosters an oriented representation of the landscape at the service of which several mediation documents apply.

\section{INDEX}

Mots-clés : planification, paysage, pédagogie, concertation, réglementation

Keywords : planning, landscape, pedagogy, consultation, regulations

\section{AUTEURS}

\section{MATHIEU GIGOT}

Mathieu Gigot est docteur en géographie et ingénieur de recherche, CJB/université d'Angers. Ses domaines de recherche portent sur la politique patrimoniale, les interactions de la géographie et du droit, la planification urbaine.

gigot.mathieu[at]gmail[dot]com

\section{ARNAUD DE LAJARTRE}

Arnaud de Lajartre est maître de conférences en droit public, $\mathrm{CJB}$ /université d'Angers. Il est responsable du programme de recherches de l'ANR sur le PLU patrimonial. Ses domaines de 
recherche portent sur les droits du patrimoine, de l'urbanisme et de l'environnement. arnaud.bernarddelajartre[at]univ-angers[dot]fr 